\title{
Comparison of Two Procedures in Application of Numerical Simulation of Shallow Water Explosion in Multi-material Media DONG $\mathrm{Qi}^{1, \mathrm{a}}$, TANG Ting $^{1, \mathrm{~b}^{*}}$, Wei Zhuo-bin ${ }^{1, c}$, ZHANG Ning ${ }^{2, d}$ \\ ${ }^{1}$ Logistics Academy,Naval University of Engineering, Tianjin 300450, China \\ 2.Naval Submarine Academy, Qingdao Shangdong 266071, China \\ adq_1990@163.com, ${ }^{\text {b }}$ kublai@126.com, 'weizhuobin@eyou.com, ${ }^{\mathrm{d}}$ 422249488@qq.com
}

Keywords: Mechanics of explosion, Multi-material media, Material dynamic behavior, Shallow water explosion, Numerical simulation, MSC.DYTRAN, LS-DYNA.

\begin{abstract}
This paper uses the LS-DYNA and MSC. DYTRAN to generate a three-dimensional numerical simulation model of shallow water explosion respectively, achieves a effective simulation of fluid-structure interaction in multi-material media, and simulates the bubble pulsation and propagation of impact wave caused by shallow water explosion. By validating the creditability of the model through comparing simulation results with the formula of empirical formula, and by analyzing the load characteristic and distribution of underwater explosive, this paper develops the following conclusions as follows. MSC. DYTRAN has a stronger capacity in boundary setting and fluid-solid coupling, therefore the bubble pulsation rate and periodic attenuation are faster and maximum radius is bigger in its simulation. There are different bubble scattering morphology on the two simulations, futher experiments are needed to verify that. The reflection effect of water bottom is stronger in LS-DYNA; the simulation effects are better for the peak pressure of shock wave loading and the specific impulse of bubble pulsation loading; the simulation is more accurate for the peak pressure of bubble pulsation loading and the specific impulse of shock wave loading in LS-DYNA.
\end{abstract}

\section{Introduction}

Shallow water explosion involves many factors including fluid-solid coupling in multi-material media, material dynamic behavior, interface and boundary effect. As a continuous process, there are specific characters and connections and interactions between the spreading process of shock wave and the loading effect of bubble pulsation process, and each process has different fitness for specific problems. Now MSC.DYTRAN and LS-DYNA are widely used in the simulation research of explosion at numerical simulation of underwater explosion[1-8]. To compare and analyse the difference and specific characters of them in shallow water explosion simulation, it is significant for further research of the damage effects that shallow water explosion has on structures with different structure characters and working conditions.

\section{Finite Element Model}

\section{FEM of LS-DYNA.}

Methods. The dimension of the model is $20 \mathrm{~m} \times 20 \mathrm{~m} \times 27 \mathrm{~m}$, with the air layer of $12 \mathrm{~m}$ high, water of $10 \mathrm{~m}$ deep, and the explosives of $100 \mathrm{~kg}$ TNT in the center of water. We divide the grid with hexahedral unit, apply multi-material ALE algorithm in the air, water and blank units and Lagrange algorithm in soil unit, and defined fluid-solid coupling through the key words *CONSTRAINED_LAGRANGE_IN_SOLID. The unit length of Euler field is $0.2 \mathrm{~m}$, and the unit quantity is 919200; the gradient grid is applied in Lagrange field with 19198 units, with settings of gravity field and hydrostatic pressure. The model boundary of Euler field is defined as OUTFLOW boundary without reflection so that flow field with infinite length can be simulated.

Materials. The model involves five materials including air, water, explosives, blanks and clay, and the explosives are described in the state equation of JWL, air in linear polynomial state equation, 
water in ylaw state equation, soil in linear elastic model with 22.4MPa of Modulus of Elasticity and 0.357 of Poisson' ratio, and blanks defined through the key words *MAT_ALE_VACUUM.

\section{FEM of MSC.DYTRAN.}

Methods. The model dimension for calculation is $30 \mathrm{~m} \times 20 \mathrm{~m} \times 27 \mathrm{~m}$. The longitudinal seperation of explosives is $10 \mathrm{~m}, 27 \mathrm{~m}$ in the $\mathrm{Z}$ direction, with air $12 \mathrm{~m}$ high, water $10 \mathrm{~m}$ deep and soil $5 \mathrm{~m}$ deep, and the distance between explosive and water bottom is $5 \mathrm{~m}$, so is that between explosives and water surface. The explosive is $100 \mathrm{~kg}$ of TNT assembled by the group. Air, water and explosives are in Euler units, soil in Lagrange units and coupled in General Coupling. The explosive centered cubic field of $1.9 \mathrm{~m} \times 1.9 \mathrm{~m} \times 1.9 \mathrm{~m}$ is the fine mesh with $0.115 \mathrm{~m}$ of unit size, and the dimension of other Euler units is $0.2 \mathrm{~m}$. The quantity of Euler field unit is 1982078. The unit dimension of Lagrange field is $0.5 \mathrm{~m}$, and the quantity of unit is 24000. Gravity fields and hydrostatic pressure are set. The boundary surface of model is composed of Dummy element with the boundary defined as Porosity.

Materials. The model involves four materials including air, water, explosives and clays, and the explosives are described in the state equation of JWL, air in $\gamma$ law state equation, water in linear polynomial state equation, soil in linear elastic model with $22.4 \mathrm{MPa}$ of Modulus of Elasticity and 0.357 of Poisson ratio.

\section{Comparison of Macro Phenomenon}

The simulation phenomena of LS-DYNA and MSC.DYTRAN are shown in Fig. 1 and Fig. 2, respectively. During the spreading phase of shock wave, their phenomena are primarily the same. After explosion, the explosion wave spreads in water, forming the underwater explosion shock wave. When the wave surface reaches the water surface and bottom, reflection comes, so water surface darkening phenomenon can be observed from the water surface.

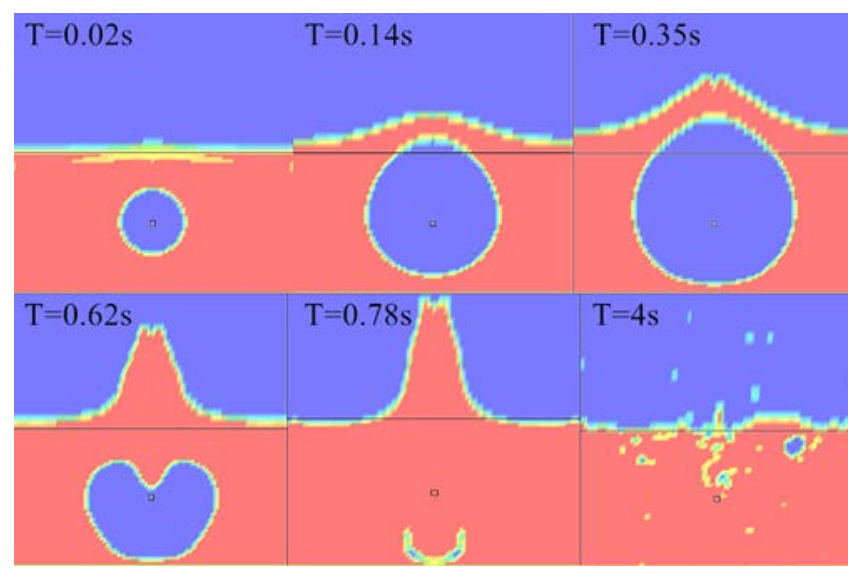

Fig. 1 Phenomenon of simulation calculation of LS-DYNA.

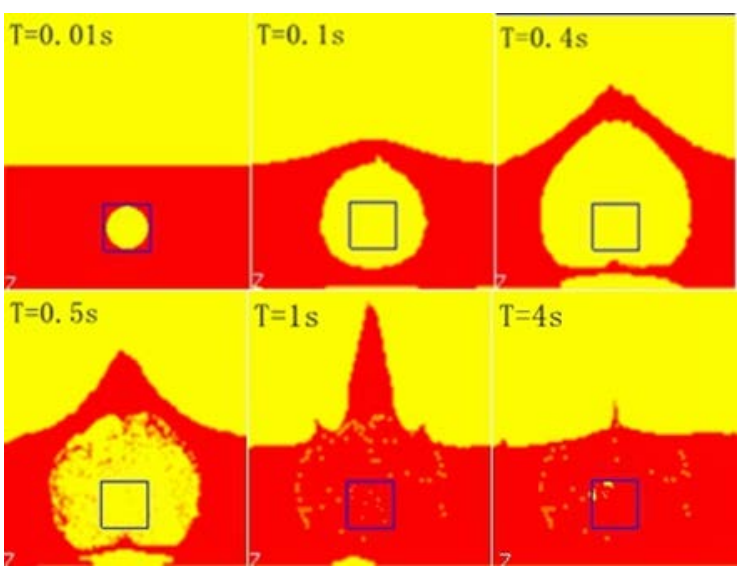

Fig. 2 Phenomenon of simulation calculation of MSC.DYTRAN.

Table 1. Comparison of bubble swelling phase.

\begin{tabular}{ccc}
\hline & Maximum radius of bubbles $(\mathrm{m})$ & Arrival time of the maximum radius(s) \\
\hline LS-DYNA & 5.92 & 0.35 \\
MSC.DYTRAN & 6.30 & 0.40 \\
\hline
\end{tabular}

As shown in Table 1, during the bubble swelling phase, the swelling rate of bubbles and the maximum radius is bigger in MSC.DYTRAN simulation because MSC.DYTRAN can simulate the real situations by setting dumb units and holes to simulate the material characteristics in the infinite flow field outside the model field, except that the two kinds of simulation can both define the pressure distribution and the seepage way. At the same time, the cavitation phenomenon at water bottom is more obvious in MSC.DYTRAN, indicating that MSC.DYTRAN can demonstrate the fluid-solid coupling state. 
When bubbles reach its biggest radius, the difference of bubble morphology development between the two simulations is great. In LS-DYNA, the bubble begins to collapse when it comes to the biggest radius while the free surface continues to rise and form a water column. Then the upper surface of bubble begins to collapse and a jet flow is produced and penetrates the bubbles to form toroidal bubbles and continues to contract. At $0.78 \mathrm{~s}$, the bubble gets to the smallest radius, rebounds, enters into an impulse period and rises to the surface. In MSC.DYTRAN, after contraction for $0.1 \mathrm{~s}$, the upper surface of bubble collapse, while the lower surface rises, then the bubble moves to the center and collapses. Bubbles collapse completely. Water rushing to the center explosives begins to splash at an angle of $45^{\circ}$ along the bottom of water column. At 2.0s, the water column falls down, and the bottom of water column falls down due to the impact. At 4s, both of the columns disappear, explosion products generally coming out of the water surface, but the water surface still moves lightly. During the whole process in MSC.DYTRAN, there are obvious water bottom perturbation and cavatition, and during the phase of bubble contracting, cavatition area develops rapidly, which results in the difference from the simulation of bubble state in LS-DYNA.

Above all, the phenomena during the shock wave spreading phase are the same for the two kinds of simulation. During the bubble swelling phase, the swelling rate is bigger, so is the maximum radius in MSC.DYTRAN. There are big differences in the bubble collapsing and contracting state between the two kinds of simulations, but the movement state of free surface is almost the same, and explosion products come out of the water surface at about $4 \mathrm{~s}$ after explosion.

\section{Load analysis and Comparison}

Load Characteristics. The Fig. 3 and 4 show the pressure time-history of the position of $5 \mathrm{~m}$ under the water surface and $5 \mathrm{~m}$ from the explosives horizentally in the explosion flow field for LS-DYNA and MSC.DYTRAN simulations, respectively. Both of the curves have three obvious phases, shock wave phase, cavatition phase and bubble pulsation phase. During the shock wave phase, the peak pressure comes up to 38.89MPa in LS-DYNA, much higher than that in MSC.DYTRAN(24.51MPa). After explosion, gases with high temperature and pressure spread out and the cavatition phase comes. Due to bubble swelling, the reflection of water surface at bottom and the boundary, the position in the curves still has obvious pressure perturbation after being sweeped by the shock wave surface, and the perturbation stops when it enters into the cavatition area. The cavatition area spreads faster in MSC.DYTRAN. At 0.1s, the position at $h=5 \mathrm{~m}$ and $\mathrm{r}=5 \mathrm{~m}$ enters into cavatition area. However, the same position enters into cavatition area at 0.165 s in LS-DYNA.

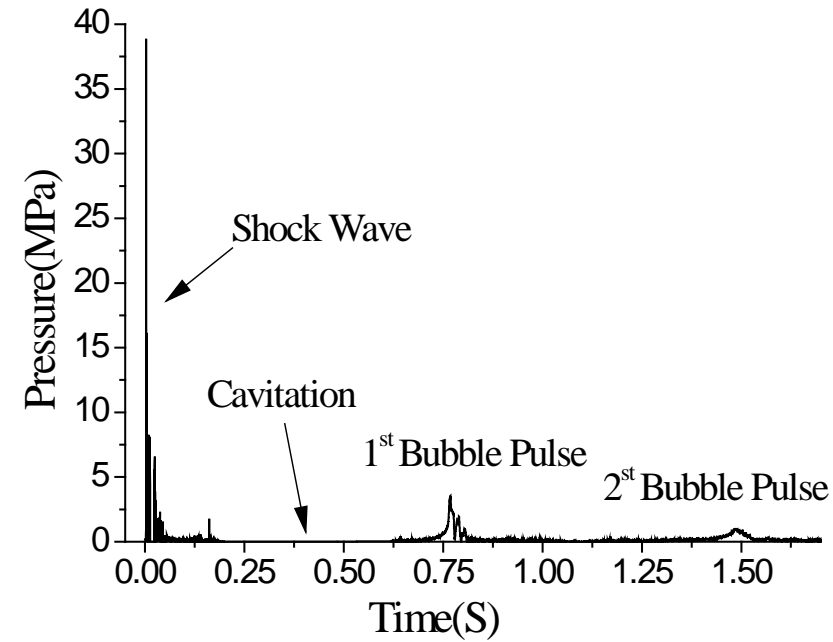

Fig. 3 Pressure time-history of LS-DYNA(h=5m, r=5m).

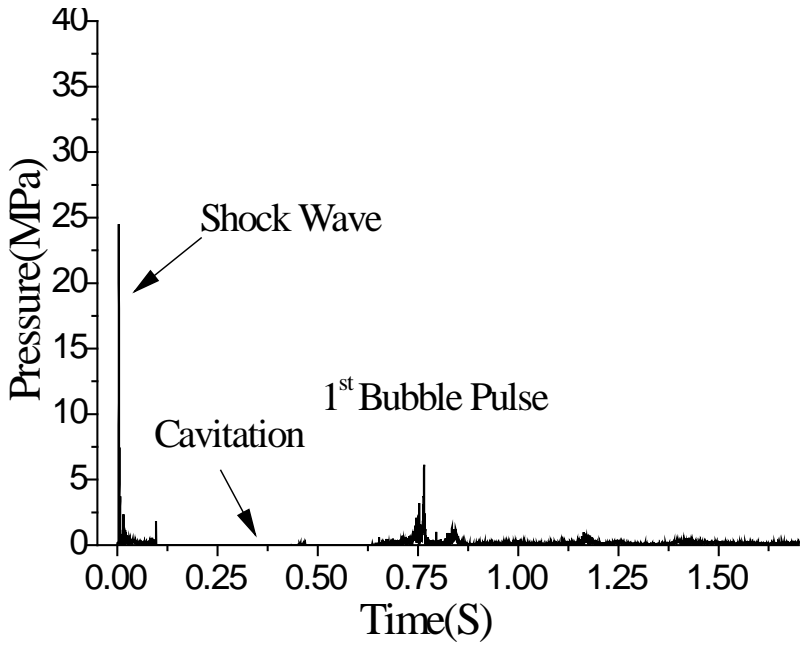

Fig. 4 Pressure time-history of MSC.DYTRAN(h=5m, $\mathrm{r}=5 \mathrm{~m})$. 
When the radius of bubbles comes up to the maximum, bubbles begin to collapse and contract. At 0.62s, the position gets out cavatition area in both LS-DYNA and MSC.DYTRAN, and then show pressure perturbation. At 0.78s, bubbles become smallest and begin to swell, and the loading of bubble swelling spread fast. At the position pressure rises obviously, which is the peak pressure of the 1st pulsation loading. The peak pressure is $6.12 \mathrm{MPa}$ in MSC.DYTRAN, much higher than 4.31MPa in LS-DYNA. But the increasing and damping of bubble loading are gentle in LS-DYNA. After the 1st pulsation of bubbles, the pressure curve become flat and the overpressure effect is not obvious, so they don't need to be compared. But we can still find that in MSC.DYTRAN the damping rate of bubble pulsation period becomes faster, so is bubble pulsation rate.

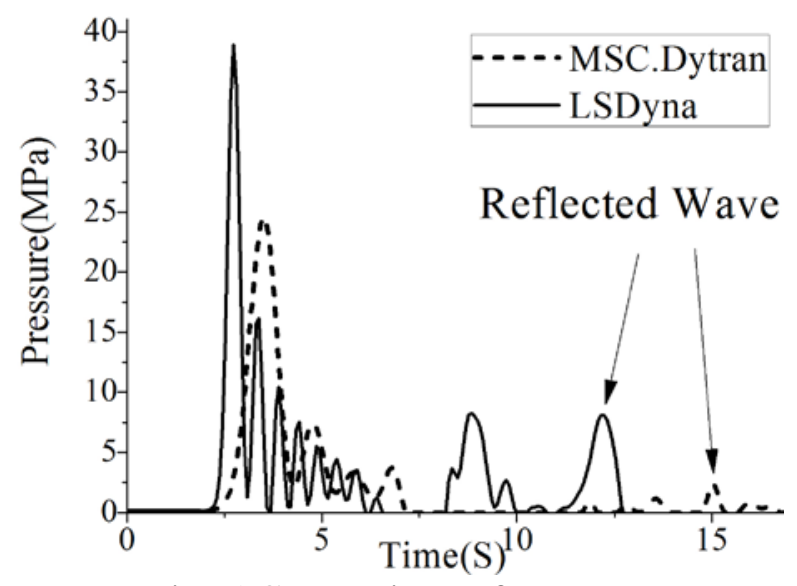

Fig. 5 Comparison of pressure time-history $(\mathrm{h}=5 \mathrm{~m}, \mathrm{r}=5 \mathrm{~m})$.

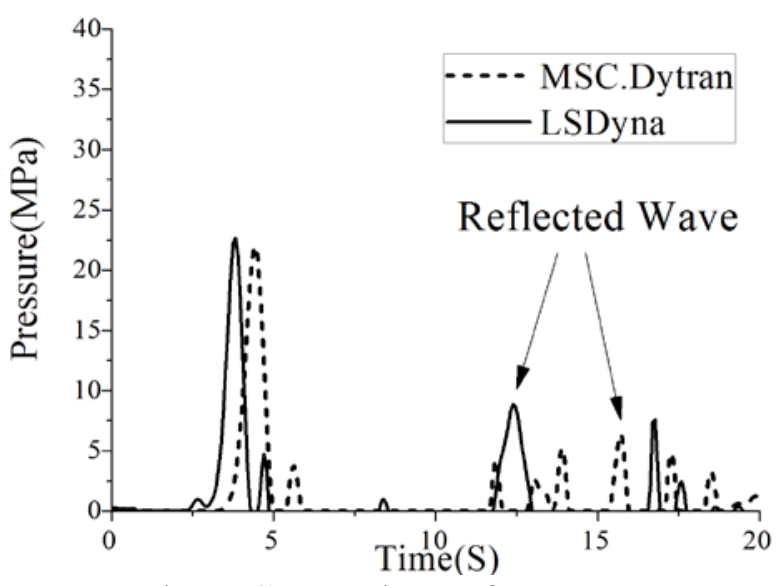

Fig. 6 Comparison of pressure time-history $(\mathrm{h}=5 \mathrm{~m}, \mathrm{r}=5 \mathrm{~m})$.

The Fig. 5 and 6 show the comparison of Pressure time-history at the position 5m under the water surface and $5 \mathrm{~m}$ from the explosives horizontally in LS-DYNA and MSC.DYTRAN, respectively. There are obvious reflections in both simulations. The closer to the bottom, the stronger the reflection, and the higher the peak pressure.

Table 2. Comparison of the parameters of the blast and reflected shock wave $(\mathrm{h}=5 \mathrm{~m}, \mathrm{r}=5 \mathrm{~m})$.

\begin{tabular}{cccccc}
\hline \multirow{2}{*}{ Software } & \multicolumn{2}{c}{ Peak pressure (MPa) } & \multirow{2}{*}{ Peak pressure ratio } & \multicolumn{2}{c}{ Arrival time of the peak pressure(s) } \\
\cline { 2 - 3 } \cline { 5 - 5 } & Blast & Reflect & & Blast & Reflect \\
\hline LS-DYNA & 38.89 & 8.10 & 0.2082 & 2.86 & 12.09 \\
MSC.DYTRAN & 24.51 & 2.37 & 0.0966 & 3.52 & 15.05 \\
\hline
\end{tabular}

Table.3 Comparison of the parameters of the blast and reflected shock wave $(\mathrm{h}=9 \mathrm{~m}, \mathrm{r}=5 \mathrm{~m})$

\begin{tabular}{cccccc}
\hline \multirow{2}{*}{ Software } & \multicolumn{2}{c}{ Peak pressure (MPa) } & \multirow{2}{*}{ Peak pressure ratio } & \multicolumn{2}{c}{ Arrival time of the peak pressure(s) } \\
\cline { 2 - 3 } \cline { 5 - 6 } & Blast & Reflect & & Blast & Reflect \\
\hline LS-DYNA & 22.67 & 8.87 & 0.3913 & 3.81 & 12.38 \\
MSC.DYTRAN & 21.91 & 6.39 & 0.2917 & 4.40 & 15.71 \\
\hline
\end{tabular}

Compared with table 2 and 3, the peak pressure at the position of $5 \mathrm{~m}$ under the water surface higher in LS-DYNA than MSC.DYTRAN, so is the reflection. The peak pressure of the reflecting shock wave is $20.82 \%$ of explosion shock wave in LS-DYNA, which is 9.66\% in MSC.DYTRAN. At the position of $9 \mathrm{~m}$ deep, the loading peak pressure is a little higher in LS-DYNA than in MSC.DYTRAN. The blast pressure of reflecting shock wave comes up to 39.13\% of explosion shock wave in LS-DYNA, higher than 29.17\% in MSC.DYTRAN. At the same conditions, in LS-DYNA, the explosion shock wave and reflecting shock wave come earlier, and the loading peak pressure is higher, the more obvious the reflection effect of the water bottom.

Comparison of Simulation Error. According to the literature [9, 10], the experience formula of the load of shock wave and the bubble pulsation in this paper under setting conditions are as followed. 


$$
\begin{aligned}
& P_{m}=52.16\left(\frac{W^{1 / 3}}{S}\right)^{1.13} \\
& I=5880 \frac{W^{0.63}}{S^{0.891}} \\
& P_{m b}=7.24 \frac{W^{1 / 3}}{S} \\
& I_{b}=3.245 \times 10^{4} \frac{W^{2 / 3}}{\mathrm{H}^{1 / 6} S}
\end{aligned}
$$

$\mathrm{W}$ is the quantity of explosives assembled; $\mathrm{H}$ is the depth of explosives under water; $\mathrm{s}$ is the spreading distance; Pm is the peak pressure of shock wave loading; I is the specific impulse of shock wave loading; Pmb is the preak pressure of bubble pulsation loading; Ib is the specific impulse of bubble pulsation; As shown in the blew table, we compare the specific loadings between the position of $5 \mathrm{~m}$ deep and $5 \mathrm{~m}$ from the explosives horizontally.

Table 4. Comparison of the characteristic parameters of the load of shock wave and bubble pulse.

\begin{tabular}{ccccc}
\hline & $\begin{array}{c}\text { Peak pressure of } \\
\text { shock wave(MPa) }\end{array}$ & $\begin{array}{c}\text { Peak pressure of } \\
\text { bubble pulse (MPa) }\end{array}$ & $\begin{array}{c}\text { Specific impulse of } \\
\text { shock wave } \\
\left(\mathrm{kNs} \cdot \mathrm{m}^{-2}\right)\end{array}$ & $\begin{array}{c}\text { Specific impulse of } \\
\text { bubble pulse } \\
\left(\mathrm{kNs} \cdot \mathrm{m}^{-2}\right)\end{array}$ \\
\hline LS-DYNA & 38.89 & 4.31 & 28.30 & 67.29 \\
DYTRAN & 24.51 & 6.12 & 25.14 & 37.4 \\
Experience & 47.96 & 6.72 & 25.50 & 106.92 \\
\hline
\end{tabular}

Compared with the empirical calculation formula, the error is smaller at simulating the peak pressure of shock wave loading and specific impulse of bubble pulsation loading in LS-DYNA, and the simulating effect is better in LS-DYNA than MSC.DYTRAN. It is more accurate to simulate the peak pressure of bubble pulsation loading and specific impulse of shock wave loading in MSC.DYTRAN. It shows that the loading rising and damping is slower in LS-DYNA than MSC.DYTRAN. The specific impulse is higher in LS-DYNA.

\section{Summary}

This paper applied LS-DYNA and MSC.DYTRAN in numerical simulations of shallow water explosion in multi-material media, respectively, compared and analyzed the phenomena, loading features, simulation error and loading distribution law etc. The conclusions are as follows.

(1) Both of the two simulations can define the pressure distribution of boundary and seepage pattern to simulate infinite flow field. Besides, MSC.DYTRAN can simulate the real situations by setting dumb units and porosity to simulate the material characteristics in the infinite flow field outside the model field. At the same time, the abilities of fluid-solid coupling are stronger and the cavatition at water bottom is more obvious in MSC.DYTRAN. The bubble swelling rate is faster and the maximum radius is bigger in MSC.DYTRAN. There are big different in bubble collapsing and contracting state in the two simulations, the upper surface of bubble collapsing in LS-DYNA and the both surface collapsing to the center in MSC.DYTRAN. The bubble pulsation rate and period damping is faster in MSC.DYTRAN. Explosion shock wave and reflection shock wave comes earlier, the loading peak pressure higher and the reflection of the water bottom more obvious in LS-DYNA.

(2) The peak pressure of shock wave loading and the specific impulse of bubble pulsation loading are effectively simulated in LS-DYNA than MSC.DYTRAN. It is more accurate to simulate the peak pressure of bubble pulsation loading and specific impulse of shock wave loading in MSC.DYTRAN. There are more obvious rising and damping process of loading in LS-DYNA than MSC.DYTRAN, so is the specific impulse. 
(3) There are many factors affecting the Water explosion, such as fluid-solid coupling in multi-material media, material dynamic behavior, interface and boundary effects. The theories and experiments of shallow water explosion are needed for further research so as to guide the selection of general procedures and the modification of models in the study of simulation and to develop the numerical simulation study.

\section{References}

[1] Grządziela, A. Modeling the effects of underwater detonation wave. Key Engineering Materials, 588(2) (2013) 175-183.

[2] Zhang, A. M., Yang, W. S., Huang, C., \& Ming, F. R.. Numerical simulation of column charge underwater explosion based on sph and bem combination. Computers \& Fluids, 71(3) (2013) 169-178.

[3] Chen, W. D., Yang, W. M., \& Zhang, F. Numerical simulation of underwater explosion based on material point method. Key Engineering Materials, 525-526(6) (2012) 109-112.

[4] Z.H. Zhang, X. Zhu, X.F Bai. The study on numerical simulation of underwater blast wave. Explsion and shock waves, 24(2) (2004) 182-188.

[5] B. Fang, X.Zhu, Z.H. Zhang, et al. Effect of parameters in numerical simulation of underwater shock wave, Journal of Harbin Engineering University. 26(4) (2005) 419-424.

[6] G.B. Huang, Y. Zhao, G.Y, Hu. Dynamic response of a typical submarine compartment subjected to underwaterexplosion. Journal of vibration and shock, 26(10) (2007) 118-125.

[7] J.H. Yuan, X. Zhu, Z.H. Zhang, et al. Numerical simulaton method study of underwater explosion load. Ship Science and Technology, 33(9) (2011) 18-23.

[8] Z.B. Wei, T. Tang, L.J. Wang. The study on shock characteristics of underwater explosion in port. Journal of Vibration and Shock, 33(6) (2014) 18-22.

[9] R. Rajendran, K. Narasimhan. Deformation and facture behaviour of plate specimens subjected to underwater explosion-a review. International Journal of Impact Engineering, 32(12) (2006) 1945-1963.

[10]Y.N. Chen. Study on damage mechanism in ship underwater explosion and structure anti-shock. Shanghai Jiao Tong University (2008). 